\title{
Unusual Fluorescent Properties of Stilbene Units and CdZnS/ZnS Quantum Dots Nanocomposites: White-Light Emission in Solution versus Light-Harvesting in Films
}

\author{
Tingchao He, Yang Gao, Yuan Gao, Xiaodong Lin, Rui Chen, Wenbo Hu, Xin Zhao, \\ Yue Wang, Hilmi Volkan Demir, Ouli Fan, Andrew C. Grimsdale, Handong Sun*
}

Nanocomposites with organic-inorganic properties represent a new field of basic research and offer prospects for many novel applications in extremely diverse fields, due to their remarkable emerging new properties and multifunctional nature. However, controllable manipulation of their fluorescent properties in different phases is still challenging, which seriously limits the related applications of nanocomposites. In this work, a convenient protocol to fabricate organic-inorganic nanocomposites composed of stilbene chromophores and CdZnS/ZnS quantum dots (ODs) pairs, with controllable fluorescent properties is presented. It is found that stable white-light emission can be achieved only in solution phase, with negligible energy transfer or reabsorption between chromophores and QDs pairs. By contrast, when the nanocomposites are deposited as blended films, they cannot give rise to white-light emission, no matter what donor/acceptor volume ratios are used. However, the blended films can exhibit near-unity efficiency (94\%) of Förster resonance energy transfer from ODs to chromophores. The underlying physical mechanisms are revealed through comprehensive steady-state and time-resolved spectroscopic analysis. This work suggests that the CdZnS/ZnS ODs/stilbene nanocomposites can be directly used for fluorescence sensors and probes in biological system as well as fundamental investigation of lightharvesting, and also sheds light on developing other new materials for artificial photosynthesis and optoelectronics.

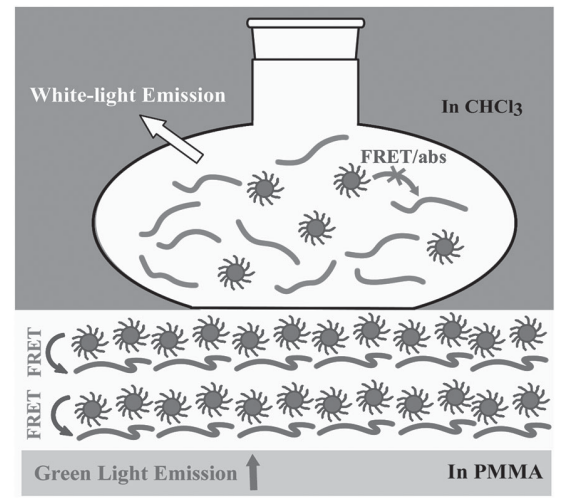

Prof. T. He, Prof. X. Lin

College of Physics Science \& Technology

Shenzhen University, Shenzhen 518060, China

Y. Gao, Prof. A. C. Grimsdale

School of Materials Science and Engineering

Nanyang Technological University

Singapore 639798, Singapore

Y. Gao, X. Zhao, Y. Wang, Prof. H. Sun

Division of Physics and Applied Physics

and Centre for Disruptive Photonic Technologies (CDPT)

School of Physical and Mathematical Sciences

Nanyang Technological University

21 Nanyang Link, Singapore 637371, Singapore

E-mail: hdsun@ntu.edu.sg

Prof. R. Chen

Department of Electrical and Electronic Engineering

South University of Science and Technology of China

Shenzhen, Guangdong 518055, P. R. China
W. Hu, Prof. O. Fan

Key Laboratory for Organic Electronics \& Information Displays (KLOEID) and Institute of Advanced Materials (IAM)

Nanjing University of Posts \& Telecommunications

Nanjing 210023, Jiangsu, China

Prof. H. V. Demir

School of Electrical and Electronic Engineering

Nanyang Technological University

Nanyang Avenue, Singapore 639798, Singapore

Prof. H. V. Demir

Department of Electrical and Electronics Engineering

Department of Physics

UNAM - Institute of Materials Science and Nanotechnology

Ankara 06800 , Turkey 


\section{Introduction}

Organic-inorganic nanocomposites combining the best features of distinct classes of different materials represent a remarkable and growing category within the world of materials science, offering great opportunities for the development of functional materials. ${ }^{[1]}$ During the last twenty years, considerable basic research has been undertaken into producing tailor-made multifunctional nanocomposites with perfect control over composition, structure, and shape. ${ }^{[2-4]}$

Semiconductor nanocrystal quantum dots (QDs) have become an important class of nanomaterials with various potential applications due to their remarkable stability and size- and shape-dependent optical and electronic properties. ${ }^{[5,6]}$ QDs possess high quantum yields with broad absorption and narrow emission bands, indicating that it is promising to use ODs to fabricate functional nanocomposites. ${ }^{[7]}$

There are a lot of prior literatures which discuss in details the energy transfer induced fluorescence enhancement between colloidal ODs and various fluorescent dyes, ${ }^{[8]}$ but reports of controllable fluorescent properties between them in different phases are scarce. For different application purposes, controlling such interactions in the inorganic-organic scaffold will be extremely important. For example, the generation of white-light emission from nanocomposites in solution is of particular interest for a wide range of applications, such as fluorescence sensors and probes in biological systems. ${ }^{[9]}$ While in the blended films of ODs and chromophores, efficient light-harvesting will be crucial for fabrication of various practical solid devices. Inspired by previous research progress, it is expected that ODs/chromophores pairs with controllable manipulation of fluorescent properties in both solution and solid phases will have more extensive applications. However, such kinds of multifunctional ODs/chromophores nanocomposites are still being explored. To realize efficient nanocomposites, it is crucial to develop chromophores that have broad tunability of fluorescence emission in different phases.

In view of the high quantum yield of blue-light emitting CdZnS/ZnS ODs and the wide tunability of fluorescence wavelength of a stilbene chromophore in different phases due to its strong intramolecular charge transfer in organic solvents and special $\pi$-stacking building in solid state, here we present the preparation of their nanocomposites in different phases. Much different from previous counterparts, with the absence of fluorescence resonance energy transfer (FRET) and reabsorption, the nanocomposites in chloroform $\left(\mathrm{CHCl}_{3}\right)$ will be especially favorable for the generation of white-light emission through direct mixture of separate blue- and orange-light emission from ODs and dye. When a chromophores/ODs/PMMA solution is deposited to form blended films, highly efficient energy transfer from CdZnS/ZnS ODs to stilbene chromophores has been achieved, though white-light emission could not be generated no matter what donor/acceptor volume ratios are used. The FRET mechanism is confirmed through comprehensive steady-state, time-resolved, and temperature dependent spectroscopic analysis.

\section{Result and Discussion}

\subsection{Optical Properties of Stilbene Chromophore: Intramolecular Charge Transfer Properties}

The detailed synthesis and characterization for stilbene chromophore are given in the Experimental Section, while its chemical structure is shown in Figure 1a. Figure $1 \mathrm{~b}$ shows the UV/vis linear absorption and fluorescence spectra of stilbene chromophore in the different mediums. From Figure $1 b$, the absorption peaks were
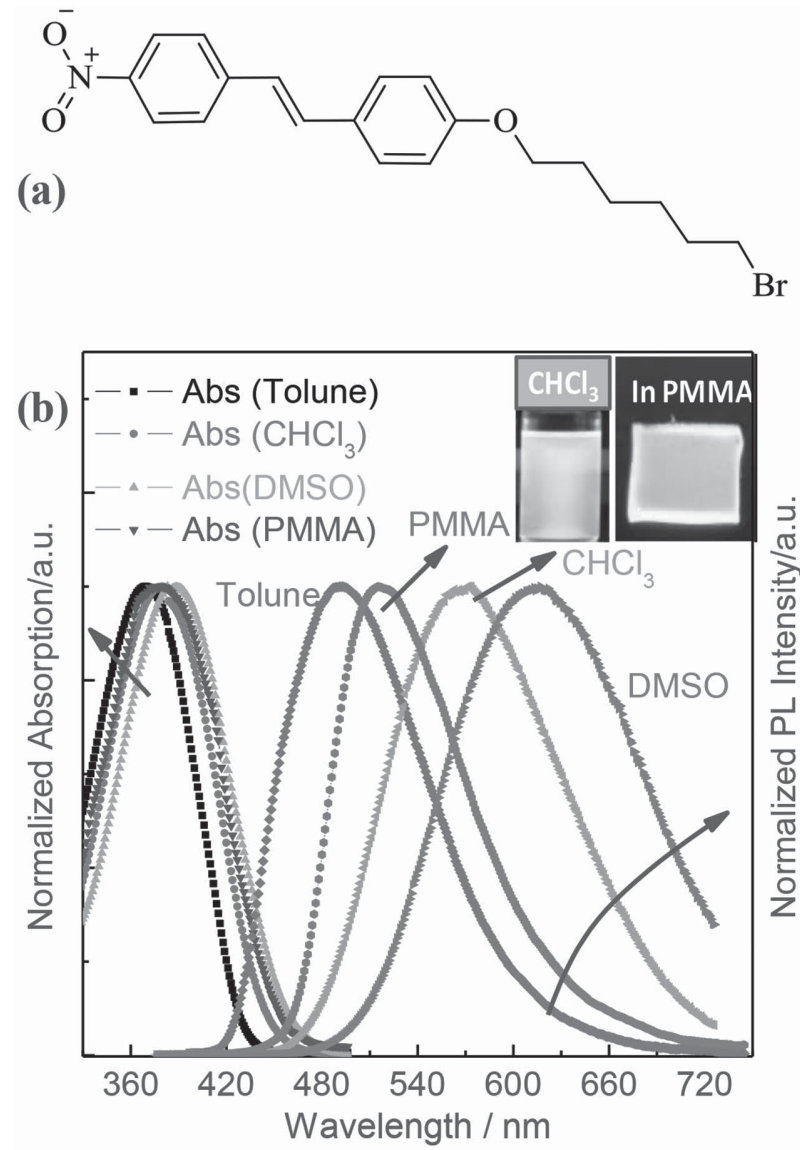

Figure 1. a) Chemical structure of stilbene chromophore studied. b) Normalized linear absorption and fluorescence spectra of the stilbene chromophore in different organic solvents and PMMA film. The insets show the photographs of fluorescence emission for the stilbene chromophore in $\mathrm{CHCl}_{3}$ and PMMA under UV excitation.

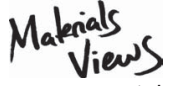

www.MaterialsViews.com 
generally red-shifted with an increase in the solvent polarity, but the extent of spectral shift was small, being merely a few nanometers. For the stilbene chromophore in PMMA film, slight broadening of the optical absorption was observed, which should be attributed to the slight aggregation effect. In sharp contrast, the fluorescence peaks of the stilbene chromophore solutions varied dramatically with solvent polarity and can be up to more than $120 \mathrm{~nm}$. It was found that in organic solvents such as toluene, $\mathrm{CHCl}_{3}$ and dimethyl sulfoxide (DMSO), the molecular emission peaks located at 490, 565, and $615 \mathrm{~nm}$, respectively. Evidently, the emission wavelength of stilbene chromophore was bathochromically with increasing solvent polarity. It was well known that organic derivatives comprising of donor and acceptor often showed such a solvatochromic effect. The similar phenomena were reported for $\mathrm{p}$-nitrostilbene derivatives and trans-alkoxynitrostilbenes, which were attributed to intramolecular charge transfer. ${ }^{[10,11]}$ Regarding the blue-shifted emission peak in PMMA film, it can be discussed in terms of the molecular packing induced crystallographic modification in solid phase. ${ }^{[11]}$ We have measured the lifetimes of the emission bands for stilbene chromophore in $\mathrm{CHCl}_{3}$ and PMMA film (Figure S1, Supporting Information). From emission lifetime emission and fractional amplitudes of them, it could be concluded that $\mathrm{CHCl}_{3}$ solution exhibited shorter weighted mean lifetime compared to the PMMA film (Table S1, Supporting Information).

\subsection{Optical Properties of CdZnS/ZnS ODs}

The composition homogeneity and size distribution of the CdZnS/ZnS ODs were analyzed by transmission electron microscopy (TEM), and the typical low-magnification and magnified images of the sample are presented in Figure 2a,b. TEM images of ODs showed a size/shape distribution. A statistical analysis on more than 150 ODs in TEM images demonstrated that the most of the as-prepared nanocrystals were dispersed with diameters of $7.5 \pm 0.3 \mathrm{~nm}$ (Figure 2c). Figure 2d shows the absorption and fluorescence spectra of colloidal and closed-packed CdZnS/ZnS ODs. For the colloidal sample, the absorption and fluorescence peaks located at 408 and $456 \mathrm{~nm}$, respectively. The observed Stokes shift of $48 \mathrm{~nm}$ and fluorescence full width at half maximum (FWHM) of $31 \mathrm{~nm}$ indicated narrow size distribution of CdZnS/ZnS ODs. For closed-packed CdZnS/ ZnS ODs, the fluorescence peak was centered at $467 \mathrm{~nm}$, about $11 \mathrm{~nm}$ red-shifted compared to the colloidal sample. The quantum confined Stark effect can be excluded as the reason for the spectral shift in closed-packed CdZnS/ZnS ODs, since only one emission band was observed. ${ }^{[12]}$ Based on the experimental results of lifetime measurements, it can be concluded that such kind of red-shift should be due to inter-ODs FRET (Figure S2, Supporting Information). Therefore, the narrow size distribution was still wide enough for the occurrence of inter-ODs FRET.

\subsection{White-Light Emission of ODs/Stilbene Chromophores in Solution}

Inspired by the fact that the fluorescent behaviors of stilbene chromophores had a strong dependence on the molecule's surrounding environment, the chromophores could be used as fluorescent sensors for solid-liquid phase change, viscosity, and temperature. ${ }^{[13]}$ Such kinds of whitelight emitting system not only preserved the surrounding environment dependent fluorescent properties of stilbene chromophore but also had bright prospect in fluorescence 
sensors and probes in biological systems. Generally, the fabrication of white-light emitting materials was not only quite complicated and costly but also involved undesired energy transfer or/and reabsorption among fluorophores. ${ }^{[14,15]}$ Therefore, alternative and quantitative approaches should be considered in this direction. It is desirable to suggest simpler, cost-effective strategies with the absence of energy transfer and reabsorption to obtain white-light emission in solution. Considering small spectral overlap between ODs and stilbene chromophore in solution, energy transfer and reabsorption may be avoided in the solution of our nanocomposites.

There are various ways toward whitelight emission in fluorescent materials. For instances, it can be realized by appropriately incorporating various fluorescent materials with the primary colors of red, green and blue, respectively, or at least two cyan colors (blue and orange emission). In view of orangelight emitting stilbene chromophore and blue-light emitting ODs in $\mathrm{CHCl}_{3}$ solution, with proper control of mixture ratio, white-light emission may be achieved from their nanocomposites. Figure $3 \mathrm{a}$ shows the normalized absorption and fluorescence spectra of ODs, stilbene chromophore as well as their spectral overlap. From the linear absorption spectra of ODs, stilbene chromophore and ODs/stilbene nanocomposite

(Figure S3, Supporting Information), we could conclude that the electronic coupling between ODs and stilbene chromophore in the mixture was negligibly small. In order to achieve white-light emission in the solution, several concentration values of dye and ODs were carefully chosen through the measurements of fluorescence spectra and the comparison of their fluorescence intensity. It was found that direct mixture of their solutions (volume ratio of stilbene chromophore/ODs) could generate white-light emission when the solutions of ODs and stilbene chromophore were prepared in a concentration of $2.5 \mu \mathrm{g} \mathrm{mL} \mathrm{m}^{-1}$ and $1 \times 10^{-5} \mathrm{M}$, respectively. The related fluorescence spectrum of the nanocomposite solution was presented in Figure $3 b$. The white-light emission was further conformed by the CIE chromaticity coordinates, which were calculated from the emission spectra of Figure 3c. The CIE coordinate of the white-light-emitting solution was $(0.30,0.31)$, which was very close to the coordinate of standard white-light $(0.33,0.33)$. The insets in Figure $3 c$ show the photographs of blue, orange, and white-light emitting ODs, stilbene chromophore, and ODs/stilbene nanocomposite in solutions under UV excitation. It was worth noting that the fluorescence intensity of stilbene chromophore (nominal acceptor) only slightly increased (Figure $3 b$ ). To confirm whether energy transfer in the nanocomposite solution was negligible, time-resolved fluorescence measurements monitoring the ODs emission at $455 \mathrm{~nm}$ were performed and the results are depicted in Figure 3d. Obviously, the donor lifetime of ODs emission almost remained unchanged, indicating weak energy transfer in the nanocomposite solution. In order to further prove that the absence of energy transfer and reabsorption in the nanocomposite solution was not due to limited neighboring stilbene chromophores around ODs, similar sample mixture with increased concentration of stilbene chromophore solution $\left(1 \times 10^{-5}\right.$ to $\left.1 \times 10^{-4} \mathrm{M}\right)$ was

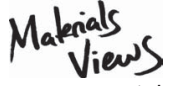

www.MaterialsViews.com 
evaluated. Again, no distinct enhancement of acceptor emission and dramatically quenched donor emission were observed, which undoubtedly confirmed that the nanocomposite exhibited weak energy transfer and reabsorption in solution (Figure S4, Supporting Information). Such kind of phenomenon was supposed by small spectral overlap of QDs emission and stilbene chromophores absorption (Figure 3a), as well as the long distance between ODs and stilbene chromophores.

Stable color quality independent of concentration (corresponding to reabsorption) is important for practical application, thus the emission properties of nanocomposites were examined under various concentrations. Through progressive dilution of the mixed solution from 1 to 24 fractions, the fluorescence spectra profiles remained unchanged over such a wide concentration range (Figure S5, Supporting Information), indicating that the color quality of nanocomposites will not be randomly disturbed by local change of solution concentration. Consequently, even though nanocomposites composed of ODs and stilbene chromophores cannot induce reabsorption and efficient energy transfer in solutions, it was extremely useful to fabricate stable white-light emission system and guarantee the reliable performance.

\subsection{FRET in Chromophores/ODs/ PMMA Blended Film}

As discussed above, compared to the case in solution, the stilbene chromophore in PMMA film was featured with much dramatic change in its physical and optical behaviors. Therefore, even though energy transfer in the nanocomposite solution was negligible, it may be expected in the blended film. In order to obtain homogenous solid films with potential light-harvesting properties, various ratios of stilbene chromophores and ODs mixture in the $\mathrm{CHCl}_{3}$ solutions were first prepared, and then PMMA was added by ultrasonic dispersion, followed by spin coating on clean substrates and drying at ambient temperature. The introduction of PMMA in the blended films can be explained as follows. PMMA is well known for excellent processability, and it has good linear transmission and excellent optical properties, which will not influence the optical properties of individual components in the blended films. On one hand, the dispersion of the chromophore into PMMA can avoid the molecular fluorescence quenching. On the other hand, the aggregation induced inter-ODs energy transfer can be weakened in PMMA host, which would facilitate the analysis of physical mechanisms of light-matter interaction between stilbene chromophore and ODs. For convenience of quantitative analysis, the solutions of ODs and stilbene chromophores were fixed with a concentration of $2.5 \mu \mathrm{g} \mathrm{mL} \mathrm{m}^{-1}$ and $1 \times 10^{-4} \mathrm{M}$, and the mass quantity ratio of QDs to PMMA was 5\%. Figure 4 a shows the spectra 
of emission and absorption for donor and acceptor. The fluorescence spectra for blended films were recorded for different donor/acceptor volume ratios (Figure $4 \mathrm{~b}$ ). The fluorescence intensity of donor (ODs) continuously decreased with the addition of more stilbene chromophores, accompanied by the increase of acceptor's green fluorescence. No matter what donor/acceptor volume ratios were used, white-light emission cannot be obtained in the blend film, due to the blue shift of acceptor's emission wavelength in solid phase. Time-resolved fluorescence measurements were used to investigate the energy transfer behavior in details. Figure 4c presents the fluorescence decay curves recorded at $460 \mathrm{~nm}$ (corresponding to ODs emission in PMMA film) for ODs in absence and in presence of acceptor. Obviously, the donor fluorescence decay in the presence of acceptor was significantly accelerated with respect to that of pure donor. In addition, the lifetime of the donor emission further decreased with increasing more fraction of acceptor in the mixture. It was thus assumed that the shortening of lifetime of donor was dominated by FRET process. For both pure donor and blended system, the fluorescence emission decayed in a multiexponential manner, which meant the energy transfer occurred with different rate constants due to the inhomogeneous nature of films (slightly different aggregation in different domain of films). ${ }^{[16]}$ The absorption of donors would contribute to the energy transfer induced fluorescence enhancement of acceptor, which can be reflected in the excitation spectra of the donor, acceptor and blend system, as indicated in Figure $4 \mathrm{~d}$. Compared to the excitation spectrum of sole acceptor, the blended film presented a distinct additional component in a region corresponding to the absorption of donor. By calculating the difference of blended system relative to pure acceptor recorded at $510 \mathrm{~nm}$, we can estimate the contribution of donor to acceptor emission in the blended system. As shown in Figure $4 d$, the difference plotted versus the wavelength resembled the excitation spectrum of donor, stressing the fact of FRET. ${ }^{[17]}$ The FRET efficiency is defined as $E=1-\tau_{\mathrm{DA}} / \tau_{\mathrm{D}}$, where $\tau_{\mathrm{DA}}$ and $\tau_{\mathrm{D}}$ are donor lifetimes with and without a neighboring stilbene chromophore. ${ }^{[18]}$ Biexponential fitting to OD decays at the wavelength of $460 \mathrm{~nm}$ in PMMA film gave satisfactory fits showing a short lifetime component of $\tau_{1}=0.34 \mathrm{~ns}$ $\left(\mathrm{A}_{1}=0.11\right)$ and a long lifetime component of $\tau_{2}=4.18 \mathrm{~ns}$ $\left(\mathrm{A}_{1}=0.89\right)$ (Table S1, Supporting Information). A substantial shortening of the OD donor decay was observed in the presence of the stilbene chromophore acceptor (Figure 4c). At highest volume ratio of stilbene chromophore/ODs $(\mathrm{V}(\mathrm{A} / \mathrm{D})=18)$, biexponential fitting yielded a major short lifetime component of $\tau_{1}=0.15 \mathrm{~ns}\left(\mathrm{~A}_{1}=0.90\right)$, and a minor long lifetime component of $\tau_{2}=1.05 \mathrm{~ns}\left(\mathrm{~A}_{2}=0.10\right)$, respectively. The fractional amplitude weighted mean lifetime of ODs in absence of stilbene chromophore $\left(\tau_{D}=3.75 \mathrm{~ns}\right)$ was considerably reduced to be $\tau_{\mathrm{DA}}=0.24 \mathrm{~ns}$ when the acceptor was present in the solution. The FRET efficiency $(E)$ was calculated as $94 \%$ for this molar ratio.

A better understanding of energy transfer occurring in the composite system can be achieved by analyzing the temperature dependence of the emission from the single components and their blended film. ${ }^{[2]}$ The normalized fluorescence spectra of donor, acceptor and blended films measured in the temperature range between 10 and $300 \mathrm{~K}$ are shown in Figure $5 \mathrm{a}-\mathrm{c}$, respectively. As indicated in Figure $5 \mathrm{a}$, the native fluorescence emission at $10 \mathrm{~K}$ from ODs, embedded in a PMMA film, was dominated by one emission band located at $466 \mathrm{~nm}$, accompanied by a weaker shoulder at $442 \mathrm{~nm}$. With increasing temperature both peaks shifted toward lower energy, and relative contribution of the peak derived from original shoulder steadily increased to dominate. Even though inter-ODs energy transfer would result in the red shift of emission as observed at low temperature, such influence can be excluded here. Considering the inter-ODs energy transfer at room temperature should be more efficient compared to that at low temperature, ${ }^{[2,19]}$ the peak derived from $466 \mathrm{~nm}$ at $10 \mathrm{~K}$ should continue to dominate the fluorescence spectra even at room temperature. Therefore, at $10 \mathrm{~K}$, the peaks at 442 and $466 \mathrm{~nm}$ should be ascribed to free exciton and the bound-exciton complexes or other native defects (instead of red shift induced by inter-ODs energy transfer), respectively. The fluorescence spectra of the bound-exciton complexes disappeared with the increasing of the temperature. Obviously, as the temperature increased, the bound excitons dissociated and transformed into free exciton. As expected, the emission profile of stilbene chromophore in PMMA film remained constant due to large bound energy of Frenkel exciton in organic molecules (Figure 5b). ${ }^{[20]}$ Interestingly, the blended film, besides the peak at $460 \mathrm{~nm}$, no distinguishable emission band for ODs was seen at any other location for all considered temperatures (Figure 5c). As the temperature increased, the peak position of fluorescence spectra still remained unchanged. Besides influence of bound excitons dissociation, due to close energy levels between defect state in ODs and excited state of stilbene chromophore, the absorbed energy was more easily transformed from the defect state of ODs to excited state of stilbene chromophore, compared to the case of energy transfer from ODs' free exciton emission state to molecular excited state (Figure $5 \mathrm{~d}$ ). As a result, the lower energy band of fluorescence emission that corresponded to bound-exciton emission was depleted, keeping the ODs emission in the blend films remaining unchanged with the increase of temperature.

For the practical application in biosensor and solid optoelectronic devices, the materials must demonstrate good photostability under laser operation. To further investigate the photostability of nanocomposites,

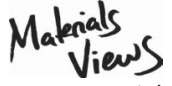

www.MaterialsViews.com
Macromol. Chem. Phys. 2016, 217, 24-31

(c) 2015 WILEY-VCH Verlag GmbH \& Co. KGaA, Weinheim

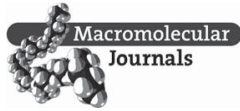



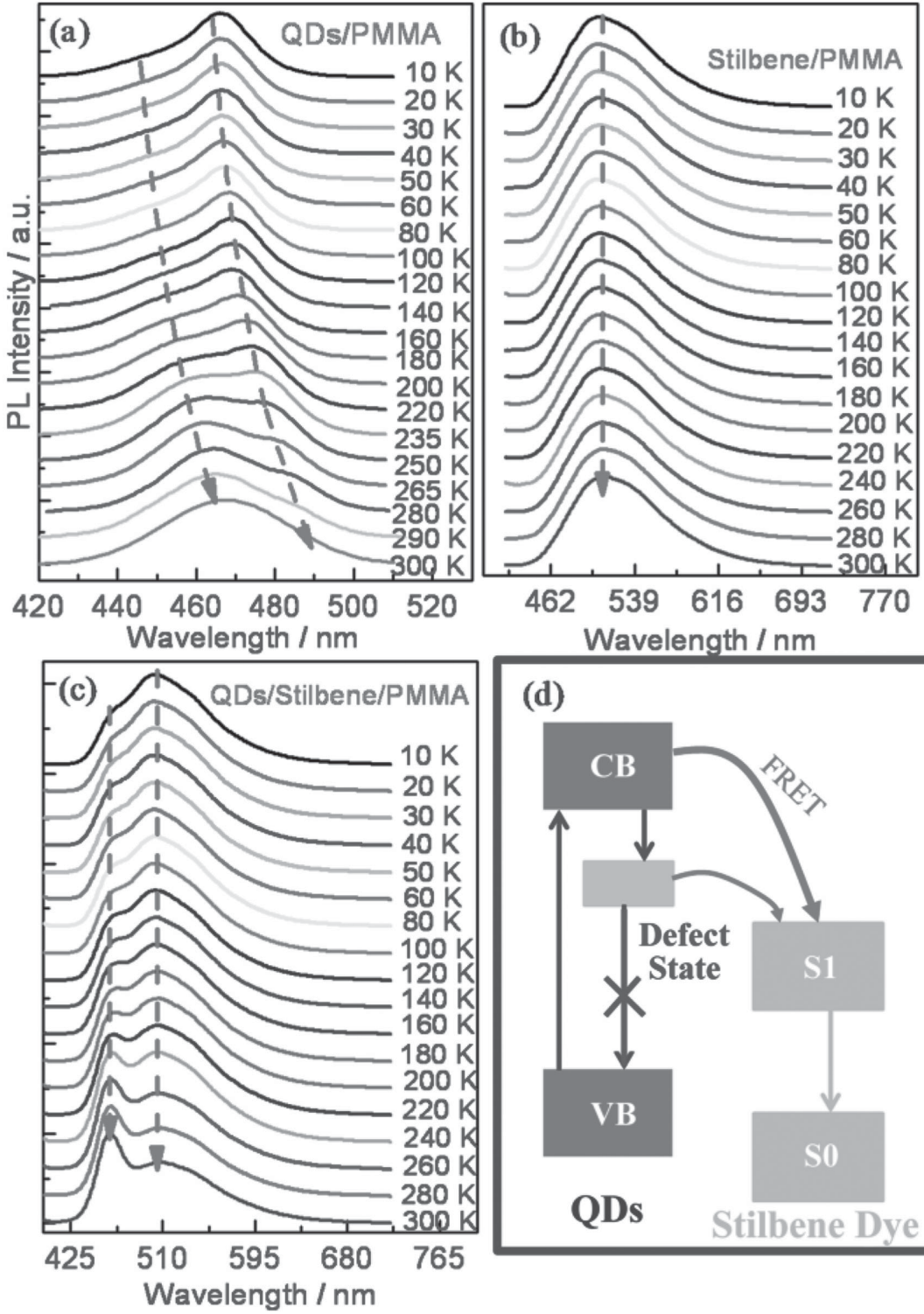

(d)

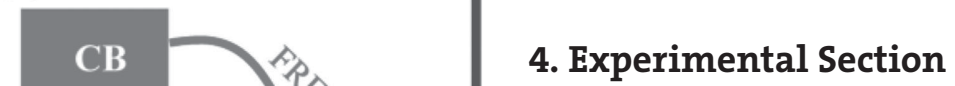

Figure 5. Temperature-dependent fluorescence spectra a) for donor, b) acceptor, and c) their blended film measured under the excitation of $325 \mathrm{~nm}$. The dashed lines follow the peak positions and are a guide for the eyes. d) The defect state of ODs mediated energy transfer in blended film. VB represents valence band, while CB represents conduction band.

bleaching tests were carried out on the both white-light emitting solution and green emitting blended film. The fluorescence emission intensity from them maintaining $100 \%$ of their initial emission even irritated at an optical intensity of $1 \mathrm{~W} \mathrm{~cm}^{-2}$ for $220 \mathrm{~min}$, indicating their excellent photostability (Figure S6, Supporting Information).

\section{Conclusions}

To sum up, the generation of white-light emission in the nanocomposite solution was much different from those materials involving energy transfer and reabsorption. In our case, ODs and stilbene chromophore emitted blue and orange fluorescence, respectively, which directly mixed and resulted in whitelight emission when suitable mixture ratio was used. Whereas in the blended film, white-light emission could not be generated, no matter what donor/ acceptor volume ratios are used. However, in this case, upon the excitation, apart from a resonant energy transfer of excitons in ODs' excited states, a recycling of trapped excitons by resonant transfer to the excited state in the stilbene chromophores was also possible, which combined to attribute to FRET induced fluorescence enhancement of

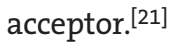

\section{Experimental Section}

Materials: Solvents, chemicals, and PMMA $\left(M_{\mathrm{w}} \gg 1900, M_{\mathrm{n}} \gg 1730\right.$, autoignition temperature $580^{\circ} \mathrm{F}$ ) were purchased from SigmaAldrich and used as received without further purification. All solvents and PMMA are analytical standard.

Stilbene Chromophore Synthesis: (E)1-((6-bromohexyl)oxy)-4-(4-nitrostyryl)benzene trans-4-nonyloxy-4'-nitrostilbene (0.50 g, $2.0 \mathrm{mmol}$ ) and 1,6-dibromohexane ( $2.44 \mathrm{~g}, 10 \mathrm{mmol}$ ) were dissolved in the mixed solvent of $\mathrm{KOH}$ ( $2 \mathrm{M}, 10 \mathrm{~mL}$, aqueous)/tetrahydrofuran $(10 \mathrm{~mL})$, with tetra-n-butylammonium bromide ( $0.68 \mathrm{~g}$. $2.1 \mathrm{mmol}$ ) as a phase transfer agent. ${ }^{[22]}$ The reaction mixture was then heated at $65^{\circ} \mathrm{C}$ overnight, before extraction with methylene dichloride (DCM) and column separation with nex:DCM 1:1 as eluting solvent. Yield: $52.5 \%$ (0.44 g, yellow powder). ${ }^{1} \mathrm{H}-\mathrm{NMR}\left(\mathrm{CDCl}_{3}\right) \delta: 8.20(2 \mathrm{H}, \mathrm{d}, \mathrm{J}=8.4$ $\mathrm{Hz}), 7.59(2 \mathrm{H}, \mathrm{d}, \mathrm{J}=8.4 \mathrm{~Hz}), 7.49(2 \mathrm{H}, \mathrm{d}, \mathrm{J}=8.4$ $\mathrm{Hz}), 7.23(1 \mathrm{H}, \mathrm{d}, \mathrm{J}=16.4 \mathrm{~Hz}), 7.01(1 \mathrm{H}, \mathrm{d}, \mathrm{J}=16.4 \mathrm{~Hz}), 6.92(2 \mathrm{H}, \mathrm{d}, \mathrm{J}=$ $8.4 \mathrm{~Hz}), 3.99(2 \mathrm{H}, \mathrm{t}, \mathrm{J}=6.4 \mathrm{~Hz}), 3.41(4 \mathrm{H}, \mathrm{m}), 1.93-1.80(4 \mathrm{H}, \mathrm{m}), 1.52$ $(2 \mathrm{H}, \mathrm{t}, 3.2 \mathrm{~Hz}) .{ }^{13} \mathrm{C} \mathrm{NMR}\left(\mathrm{CDCl}_{3}\right) \delta: 159.77,146.40,144.34,132.97$, $128.84,128.44,126.49,124.17,124.00,114.88,67.86,33.93$, 32.75, 29.57, 28.01, 25.43. MALDI-TOF-MS: 404.82 (Calculated: $\mathrm{C}_{20} \mathrm{H}_{22} \mathrm{BrNO}_{3}, 404.30$ ). Melt point: $86.7^{\circ} \mathrm{C}$. The detailed synthesis information can be found in the Supporting Information.

CdZnS/ZnS QDs Synthesis: Blue-emitting ODs were synthesized via standard high temperature approach in noncoordinating solvent. Cadium oxide and zinc acetate were dissolved in oleic acid, and sulfur in 1-Octadecene was injected into the aforementioned mixture at $310^{\circ} \mathrm{C}$, with the temperature maintained for $12 \mathrm{~min}$. For outmost zinc sulfide growth, the sulfur injection dose was 
prepared by dissolving elementary sulfur in oleic acid, and the dose was injected dropwisely. The reaction lasted three hours. Compared with other synthetic protocols for blue emitting $\mathrm{Cd}$ based colloidal quantum dots, the oleic acid ligands that passivate the ODs surface demonstrated much stronger binding and better passivation effect. The blue-emitting ODs prepared based on this protocol, due to long shelling duration, were large in size and have thick shells, which gave rise to high absorption cross section and fluorescence quantum yield, as well as excellent photostability. The blue-emitting ODs in solution had a fluorescence quantum yield up to $85 \%$.

Instruments: High-resolution transmission electron microscopy (HRTEM) images were taken by using a JEOL JEM-2010. The morphologies of the samples were characterized by the field emission scanning electron microscope (JEOL-6700F model). The absolute fluorescence quantum yields of all the samples were measured using integrating spheres (GSTM-VGMS-400).

Measurements of UV-vis Absorption, Fluorescence Emission, and Fluorescence Lifetime. UV-vis absorption spectra were measured with a Shimadzu UV-vis spectrophotometer. The fluorescence spectra of the samples were collected from the samples, and then the signals were dispersed by a $750 \mathrm{~mm}$ monochromator combined with suitable filters and detected by a photomultiplier tube (Hamamatsu R928) using a standard lock-in amplifier technique. Excitation spectra were obtained using a $450 \mathrm{~W}$ xenon lamp monochromated with a double CzernyTurner spectrometer (GEMINI 180) whose excitation intensity was precorrected. The emission lifetimes were obtained from time-correlated single photon counting (TCSPC) technique, with a resolution of 10 ps (PicoQuant PicoHarp 300). The second harmonic generation of Titanium sapphire laser (Chameleon, Coherent Inc.) operating at $360 \mathrm{~nm}$ (100 fs, $80 \mathrm{MHz}$ ) was used as the excitation source. The temperature dependent fluorescence measurements were performed between 10 and $300 \mathrm{~K}$ within a helium closed-cycle cryostat.

\section{Supporting Information}

Supporting Information is available from the Wiley Online Library or from the author.

Acknowledgements: T.H. and Y.G. contributed equally to this work. This work was supported by the Natural Science Foundation of China (Grant Nos. 11404219 and 11404161), Natural Science Foundation of Guangdong Province (Grant No. 2014A030313552), Strategic Emerging Industry Development Special Fund of Shenzhen (JCYJ20150324141711613), and the startup fund from SUSTC and national 1000 plan for young talents. This research was also supported by the Singapore National Research Foundation through the Competitive Research Progamme(CRP) under Project No. NRFCRP5-2009-04 and the Singapore Ministry of Education through the Academic Research Fund under Project No. MOE2011-T3-1-005(Tier 3).

Received: September 7, 2015; Published online: October 26, 2015; DOI: $10.1002 /$ macp. 201500350

Keywords: CdZnS/ZnS quantum dots; energy transfer; fluorescent nanocomposites; stilbene; white-light emission
[1] C. Sanchez, P. Belleville, M. Popalld, L. Nicoleab, Chem. Sov. Rev. 2011, 40, 696.

[2] B. Guzelturk, P. L. Hernandez-Martinez, V. K. Sharma, Y. Coskun, V. Ibrahimova, D. Tuncel, A. O. Govorov, X. W. Sun, O. Xiong, H. V. Demir, Nanoscale 2014, 6, 11387.

[3] Y. Dang, H. Li, Y. Wu, ACS Appl. Mater. Interfaces 2011, 4, 1267.

[4] N. Bouchonville, A. L. Cigne, A. Sukhanova, M. Molinari, I. Nabiev, Laser Phys. Lett. 2013, 10, 085901.

[5] X. Peng, L. Manna, W. Yang, J. Wickham, E. Scher, A. Kadavanich, A. P. Alivisatos, Nature 2000, 404, 59.

[6] Y. Wang, K. S. Leck, V. D. Ta, R. Chen, V. Nalla, Y. Gao, T. C. He, H. V. Demir, H. D. Sun, Adv. Mater. 2015, 27, 169.

[7] a) E. H. Cho, B. Kim, S. Jun, J. Lee, D. H. Park, K. Lee, J. Kim, J. Kim, J. Joo, Adv. Func. Mater. 2014, 24, 3684; b) G. Jiang, A. S. Susha, A. A. Lutich, F. D. Stefani, J. Feldmann, A. L. Rogach, ACS Nano 2009, 3, 4127; c) M. Locritani, Y. Yu, G. Bergamini, M. Baroncini, J. K. Molloy, B. A. Korgel, P. Ceroni, J. Phys. Chem. Lett. 2014, 5, 3325; d) M. Cargnello, B. T. Diroll, E. A. Gaulding, C. B. Murray, Adv. Mater. 2014, 26, 2419; e) K. H. Ku, M. P. Kim, K. Paek, J. M. Shin, S. Chung, S. G. Jang, W. Chae, G. Yi, B. J. Kim, Small 2013, 9, 2667; f) Y. Zhao, P. Schapotschnikow, T. Skajaa, T. J. H. Vlugt, W. J. M. Mulder, C. de M. Donegá, A. Meijerink, Small 2014, 10, 1163.

[8] a) J. E. Halpert, J. R. Tischler, G. Nair, B. J. Walker, W. Liu, V. Bulović, M. G. Bawendi, J. Phys. Chem. C 2009, 113, 9986; b) B. J. Walker, G. P. Nair, F. L. Marshall, V. Bulović, M. G. Bawendi, J. Am. Chem. Soc. 2009, 131, 9624; c) B. J. Walker, V. Bulović, M. G. Bawendi, Nano Lett. 2010, 10, 3995; d) O. Zhang, T. Atay, J. R. Tischler, M. S. Bradley, V. Bulović, A. V. Nurmikko, Nat. Nanotechnol. 2007, 2, 555; e) A. R. Clapp, I. L. Medintz, B. R. Fisher, G. P. Anderson, H. Mattoussi, J. Am. Chem. Soc. 2005, 127, 1242; f) A. S. Medel, J. Mallet, J. Brochon, A. Feltz, M. Oheim, W. J. Parak, Nano Lett. 2007, 7, 2613.

[9] X. Zhang, D. Görl, F. Würthner, Chem. Commun. 2013, 49, 8178.

[10] F. Gao, L. Yang, L. Yang, H. Li, S. Zhang, J. Fluoresc. 2010, $20,353$.

[11] C. Chen, T. Hinoue, I. Hisaki, M. Miyata, N. Tohnai, Tetrahedron Lett. 2013, 54, 1649.

[12] U. T. D. Thuy, P. T. Thuy, N. O. Liem, L. Li, P. Reiss, Appl. Phys. Lett. 2010, 96, 073102.

[13] M. Han, Y. Tian, Z. Yuan, L. Zhu, B. A. Ma, Angew. Chem. Int. Ed. 2014, 53, 11088.

[14] C. Romero-Nieto, S. Durben, I. M. Kormos, T. Baumgartner, Adv. Funct. Mater. 2009, 19, 3625.

[15] X. Wang, X. Yan, W. Li, K. Sun, Adv. Mater. 2012, 24, 2742.

[16] P. T. K. Chin, R. A. M. Hikmet, S. C. J. Meskers, R. A. Janssen, Adv. Funct. Mater. 2007, 17, 3829.

[17] T. He, R. Chen, Z. B. Lim, D. Rajwar, L. Ma, Y. Wang, Y. Gao, A. C. Grimsdale, H. Sun, Adv. Opt. Mater. 2014, 2, 40.

[18] J. R. Lakowicz, Principles of Fluorescence Spectroscopy, 3nd ed., Springer, Germany 2006.

[19] A. A. R. Neves, A. Camposeo, R. Cingolani, D. Pisignano, Adv. Funct. Mater. 2008, 18, 751.

[20] S. Blumstengel, S. Sadofev, C. Xu, J. Puls, F. Henneberger, Phys. Rev. Lett. 2006, 97, 237401.

[21] a) T. Franzl, T. A. Klar, S. Schietinger, A. L. Rogach, J. Feldmann, Nano Lett. 2004, 4, 1599; b) T. C. He, Y. Gao, R. Chen, L. Ma, D. Rajwar, Y. Wang, A. C. Grimsdale, H. Sun, Macromolecules 2014, 47, 1316.

[22] a) S. Jin, M. Wübbenhorst, J. Turnhout, W. Mijs, Macromol. Chem. Phys. 1996, 197, 4135; b) F. Tittarelli, P. Masson, A. Skoulios, Liquid Cryst. 1997, 22, 721.

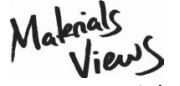

www.MaterialsViews.com 